\title{
MULTIPLE SOLUTIONS FOR SINGULARLY PERTURBED SEMILINEAR ELLIPTIC EQUATIONS IN BOUNDED DOMAINS
}

\author{
MICHINORI ISHIWATA
}

Received 17 May 2003

We are concerned with the multiplicity of solutions of the following singularly perturbed semilinear elliptic equations in bounded domains $\Omega:-\varepsilon^{2} \Delta u+a(\cdot) u=u|u|^{p-2}$ in $\Omega, u>0$ in $\Omega, u=0$ on $\partial \Omega$. The main purpose of this paper is to discuss the relationship between the multiplicity of solutions and the profile of $a(\cdot)$ from the variational point of view. It is shown that if $a$ has a "peak" in $\Omega$, then (P) has at least three solutions for sufficiently small $\varepsilon$.

\section{Main theorem}

We are concerned with the multiplicity of solutions for the following singularly perturbed semilinear elliptic equations:

$(\mathrm{P})_{\mathcal{\varepsilon}}$

$$
\begin{gathered}
-\varepsilon^{2} \Delta u+a(\cdot) u=u|u|^{p-2} \quad \text { in } \Omega, \\
u>0 \quad \text { in } \Omega, \\
u=0 \quad \text { on } \partial \Omega,
\end{gathered}
$$

where $\varepsilon \in \mathbb{R}^{+}, \Omega \subset \mathbb{R}^{N}(N \geq 1)$ is a bounded domain, $p \in\left(2,2^{*}\right)\left(2^{*}\right.$ denotes the critical exponent of the Sobolev embedding $H^{1}(\Omega) \hookrightarrow L^{p}(\Omega)$ given by $2^{*}=2 N /(N-2)$ if $N \geq 3$ and $2^{*}=+\infty$ if $\left.N=1,2\right)$. The main purpose of this paper is to discuss the relationship between the multiplicity of solutions of $(\mathrm{P})_{\varepsilon}$ and the shape of the profile of $a(x)$ when $\varepsilon$ is small. In order to characterize the topological feature of $a(x)$, we introduce the following condition $(\mathrm{A})_{K, r, \underline{c}, \bar{c}, \delta, \rho}$ for positive numbers $r, \underline{c}, \bar{c}, \delta, \rho$, and a closed subset $K$ of $\Omega$.

$(\mathrm{A})_{K, r, c, \bar{c}, \delta, \rho}: a(x) \in C(\Omega) \cap L^{N / 2}(\Omega)$ and the following conditions (i), (ii), (iii), and (iv) are satisfied:

(i) $\partial K$ is homotopically equivalent to $S^{N-1}, B(0, \rho)=\left\{x \in \mathbb{R}^{N} ;|x|<\rho\right\} \subset K$ and $(\partial K)_{r}=\left\{x \in \mathbb{R}^{N} \mid \operatorname{dist}(x, \partial K) \leq r\right\} \subset \Omega$,

(ii) $\inf _{\Omega} a(x) \geq \underline{c}$,

(iii) $\max _{\overline{B(0, \rho)}} a(x)>\bar{c}$,

(iv) $\max _{(\partial K)_{r}} a(x) \leq \underline{c}+\delta<\bar{c}$. 
Roughly speaking, the condition above implies that $a(x)$ has a "peak" in $K$ (condition (iii)), the value of $a(x)$ on $\partial K$ is uniformly less than the level of the peak (condition (iv)), and $\partial K$ forms a set which surrounds the peak and is homotopically equivalent to $S^{N-1}$ (condition (i)).

Then our main result reads as follows.

Theorem 1.1. For any positive numbers $\rho, \underline{c}, \bar{c}$ with $\underline{c}<\bar{c}$, there exists a (sufficiently small) positive number $\delta$ depending on $\rho, \underline{c}, \bar{c}$, and $\Omega$ such that if a satisfies $(\mathrm{A})_{K, r, c, \bar{c}, \delta, \rho}$ for some $r>0$ and a closed subset $K$ of $\Omega$, then there exists a positive number $\varepsilon_{0}$ so that $(\mathrm{P})_{\varepsilon}$ admits at least three solutions for all $\varepsilon \in\left(0, \varepsilon_{0}\right]$.

We give some examples of the function $a(\cdot)$ satisfying the assumption of Theorem 1.1.

Example 1.2. Let $\Omega$ be a bounded domain which contains the closure of $B(0, R)$. Let $a \in C(\bar{\Omega})$ and assume that there exist some positive numbers $\rho, \underline{c}, \bar{c}$ with $\underline{c}<\bar{c}$ and a closed subset $L$ of $B(0, R)$ such that

$$
\begin{gathered}
B(0, \rho) \subset L, \\
a(\cdot)=\underline{c} \quad \text { in } \Omega \backslash L, \\
\underline{c}=\min _{L} a(\cdot)<\bar{c}<\frac{\min }{B(0, \rho)} a(\cdot) .
\end{gathered}
$$

Take $R_{1}(<R)$ and $r>0$ such that

$$
L \subset B\left(0, R_{1}\right), \quad\left(\partial B\left(0, R_{1}\right)\right)_{r} \subset B(0, R) \backslash L .
$$

Then $a(\cdot)$ satisfies $(\mathrm{A})_{K, r, \underline{c}, \bar{c}, \delta, \rho}$ for $K=\overline{B\left(0, R_{1}\right)}, \delta \in(0, \bar{c}-\underline{c})$, and $r, \underline{c}, \bar{c}, \rho$ above.

Example 1.3. Let $\Omega$ be a bounded domain containing $\overline{B(0, \rho)}$ for some $\rho>0$ with smooth boundary $\partial \Omega$ which is homeomorphic to $S^{N-1}$. Let $a \in C(\bar{\Omega})$ and assume that there exist some positive numbers $\underline{c}, \bar{c}$ with $\underline{c}<\bar{c}$ such that

$$
\begin{gathered}
\underline{c}=\inf _{\Omega} a(\cdot)<\bar{c}<\max _{\overline{B(0, \rho)}} a(\cdot), \\
\underline{c}=a(x) \quad \forall x \in \partial \Omega .
\end{gathered}
$$

Then it is easy to see that for any small $\delta>0$, there exists a number $r>0$ such that

$$
\begin{gathered}
\partial(\Omega)_{2 r}^{-} \text {is homeomorphic to } S^{N-1}, \\
B(0, \rho) \subset(\Omega)_{2 r}^{-}, \\
\max _{\left(\partial(\Omega)_{2 r}^{-}\right)_{r}} a(x) \leq \underline{c}+\delta \quad(<\bar{c}),
\end{gathered}
$$

where $(\Omega)_{2 r}^{-}=\{x \in \Omega ; \operatorname{dist}(x, \partial \Omega) \geq 2 r\}$. Hence $a(\cdot)$ satisfies $(\mathrm{A})_{K, r, c, \bar{c}, \delta, \rho}$ for $K=(\Omega)_{2 r}^{-}$ and $r, \underline{c}, \bar{c}, \delta, \rho$ above.

Note that in this case, $a(\cdot)$ may not possess any global (local) minimum in $\Omega$. 
Remark 1.4. (1) It would be a routine work to prove that $(\mathrm{P})_{\varepsilon}$ admits at least one solution $u_{0}$ (the "ground state solution") for all $\varepsilon \in(0, \infty)$ with the aid of the well-known Mountain Pass lemma and the compactness of the Sobolev embedding $H^{1} \hookrightarrow L^{p}$. However, one cannot expect in general the existence of multiple solutions. Indeed, for example, when $a(x) \equiv 1$ and $\Omega=$ ball, the uniqueness result for sufficiently small $\varepsilon$ is known (Dancer [6]). Theorem 1.1 says that immediately after $a(x)$ is perturbed to have a "peak", other solutions $u_{1}, u_{2}$ should appear even if the perturbation is very small (in the radial case, $u_{0}$ and one of the $u_{1}$ and $u_{2}$, say $u_{1}$, may be geometrically equivalent to each other, i.e., they may coincide via rotation in $\mathbb{R}^{N}$, so one gets at least two geometrically distinct solutions, $u_{0} \sim u_{1}$ and $u_{2}$ ). This "generation of higher energy solution" is a consequence of the change of topology of some level sets of the functional associated to $(\mathrm{P})_{\varepsilon}$ caused by the nontrivial shape of $a(x)$. It is the purpose of this paper to discuss the effect of this change of topology on the multiplicity of solutions.

(2) It is already known that if $a \in C(\Omega)$ and the global minimum set of $a(x), a_{\min }=$ $\left\{x \in \Omega ; a(x)=\min _{y \in \Omega} a(y)\right\}$, is homotopically equivalent to $S^{N-1}$, then there exist at least cat $a_{\min }=\operatorname{cat} S^{N-1}=2$ solutions for small $\varepsilon$ (here cat means the Ljsternik-Schnirelman category, see Definition 3.3 below). It should be noted that our assumption $(\mathrm{A})_{K, r, c, \bar{c}, \delta}$ does not require that $a(x)$ should have a global minimum set in $\Omega$ as is stated in Example 1.3 , but requires that "nearly" global minimum set of $a(x)$ should contain the set $\partial K$ which is homotopically equivalent to $S^{N-1}$.

(3) Another type of multiplicity result for $-\Delta u+u=a(x) u+f(x)$ in $\mathbb{R}^{N}$, based on an argument similar to ours, is discussed in Adachi and Tanaka [1].

\section{Known results and notation}

2.1. Known results. The interest in $(\mathrm{P})_{\mathcal{\varepsilon}}$ arises from several physical and mathematical backgrounds.

In the physical context, $(\mathrm{P})_{\varepsilon}$ can be regarded as a (reduced) nonlinear Schrödinger equation and small parameter $\varepsilon$ corresponds to the Dirac constant $\hbar$.

It is well known that when $\hbar$ can be well-approximated by 0 (this approximation is called "semiclassical approximation"), quantum mechanical equation may have a solution corresponding to a "semiclassical" state, concentrating around a classical mechanical equilibrium. It is also well known that the classical equilibrium is often given as the point which minimizes the potential energy.

So it is reasonable to expect that for small $\varepsilon,(\mathrm{P})_{\mathcal{\varepsilon}}$ has a semiclassical solution concentrating around a point which attains the minimum of the energy potential $a(x)$. Hence the structure of $a_{\min }=\left\{x \in \Omega \mid a(x)=\min _{y \in \Omega} a(y)\right\}$, the minimum set of $a(x)$, may play a significant role for the existence and the multiplicity of solutions of $(\mathrm{P})_{\varepsilon}$.

In the mathematical context, $(\mathrm{P})_{\varepsilon}$ can be regarded as a typical model exemplifying the following feature. In many semilinear elliptic problems including small parameters (e.g., semilinear elliptic equations involving the critical exponent [10], stationary CahnHilliard equation [2], Ginzburg-Landau equation [3]), it is commonly observed that if the parameter is small enough, then the existence and multiplicity of solutions are controlled by the finite-dimensional object. As for singularly perturbed equations, del-Pino and Felmer [7, 8] and Cingolani and Lazzo [5] obtain the following result. 
Proposition 2.1 (effect of weight function, del Pino and Felmer [7]). Assume that $a(x)$ is a locally Hölder continuous function and $\Lambda$ is a bounded set compactly contained in $\Omega$. Also assume that there exists a positive constant $\alpha$ such that $\inf _{x \in \Omega} a(x) \geq \alpha$ and, $\min _{\partial \Lambda} a(x)>$ $\inf _{\Lambda} a(x)$. Then for sufficiently small $\varepsilon,(\mathrm{P})_{\varepsilon}$ admits a solution $u_{\varepsilon}$, which concentrates to a point in $\Lambda$ where the minimum of $a(x)$ is attained as $\varepsilon \rightarrow 0$.

Proposition 2.2 (effect of weight function, del Pino and Felmer [8]). Assume that $a(x) \in$ $C^{1}(\Omega)$ and there exists a positive constant $\alpha$ such that $\inf _{x \in \Omega} a(x) \geq \alpha$. Let $x_{0} \in \Omega$ be a "topologically nontrivial critical point" of $a(x)$ (this class of critical points includes the local minimum, the local maximum, and the saddle point of $a(x)$. For the precise definition, see [8, page 249]). Then for sufficiently small $\varepsilon,(P)_{\varepsilon}$ admits a solution $u_{\varepsilon}$, which concentrates to $x_{0}$ as $\varepsilon \rightarrow 0$.

Proposition 2.3 (effect of the topology of $a_{\min }$, Cingolani and Lazzo [5]). Assume that $a(x) \in C\left(\mathbb{R}^{N}\right)$ and $\lim _{|x| \rightarrow \infty} a(x)=a_{0}>\min _{x \in \mathbb{R}^{N}} a(x)>0$. Then for sufficiently small $\varepsilon,(\mathrm{P})_{\varepsilon}$ admits at least cat $a_{\min }$ solutions. Here cat $a_{\min }$ denotes the Ljsternik-Schnirelman category of $a_{\min }$ (see Definition 3.3 below).

The finite-dimensional objects referred to above in Propositions 2.1, 2.2, and 2.3 are the local minimum set (point) of $a(x)$, the "topologically nontrivial" critical set (point) of $a(x)$, and the global minimum set of $a(x)$, respectively.

Our problem $(\mathrm{P})_{\varepsilon}$ also bears some interesting aspect in the context of the so-called "variational problem with lack of compactness". As stated in Section 1, for problem $(\mathrm{P})_{\varepsilon}$ with bounded $\Omega$, one can easily find that there exists at least one solution of $(\mathrm{P})_{\varepsilon}$, the ground state solution, with the aid of the compactness of the Sobolev embedding $H^{1}(\Omega) \hookrightarrow L^{p}(\Omega)$. On the other hand, in the case of unbounded $\Omega$, the situation changes drastically. That is, $(\mathrm{P})_{\varepsilon}$ may not have a ground state solution. From the point of view of the variational analysis, this nonexistence is caused by the breakdown of the PalaisSmale condition for the functional associated with $(\mathrm{P})_{\varepsilon}$ due to the fact that the Sobolev embedding $H^{1}(\Omega) \hookrightarrow L^{p}(\Omega)$ is no longer compact for unbounded $\Omega$.

Even though we are concerned with $(\mathrm{P})_{\varepsilon}$ in bounded domains (the original problem), the analysis of $(\mathrm{P})_{\varepsilon}$ in $\mathbb{R}^{N}$ with some weight function determined by $a(x)$ (the limiting problem) plays a crucial role in investigating the multiple existence of solutions of $(\mathrm{P})_{\mathcal{E}}$. That is, the lack of compactness of the variational problem associated with $(\mathrm{P})_{\mathcal{\varepsilon}}$ in unbounded domains with (suitably chosen) weight functions causes the multiplicity of solutions of $(\mathrm{P})_{\varepsilon}$ in bounded domains. In other words, for small $\varepsilon,(\mathrm{P})_{\varepsilon}$ can be treated as a problem on "almost unbounded domains" with Palais-Smale condition.

Applying propositions above to our problem, we find that the following facts hold true.

(1) If $a_{\min }=\partial K\left(\simeq S^{N-1}\right) \subset \Omega$, then Proposition 2.3 assures the existence of at least cat $a_{\min }=\operatorname{cat} \partial K=\operatorname{cat} S^{N-1}=2$ solutions of $(\mathrm{P})_{\varepsilon}$ for small $\varepsilon$.

(2) Suppose that $a(x)$ has a global maximum point in $\Omega$ and $a(x) \in C^{1}(\Omega)$. Then Proposition 2.2 implies that there exists at least one solution of $(\mathrm{P})_{\varepsilon}$ for small $\varepsilon$, which concentrates to the global maximum point of $a(x)$ as $\varepsilon \rightarrow 0$.

As is pointed out in Section 1 (Example 1.3 and Remark 1.4), in our Theorem 1.1, we need not assume $a_{\min }=\partial K$ nor $a(x) \in C^{1}(\Omega)$. 
Moreover, our argument here relies on the comparison of variational structures of the original and the limiting problem with nontrivial weight function and, seems somewhat different from those in $[5,7,8]$.

2.2. Notation. We here fix the notation frequently used in this paper.

Let $\omega$ be a domain of $\mathbb{R}^{N}$, and we use the following notation.

(i) $M_{p}(\omega):=\left\{u \in H_{0}^{1}(\omega) ;\left\|u^{+}\right\|_{L^{p}(\omega)}=1\right\}$ where $u^{+}(x):=\max (0, u(x))$.

(ii) $I_{\varepsilon, a, \omega}(u):=\int_{\omega}\left(\varepsilon^{2}|\nabla u|^{2}+a u^{2}\right) d x$.

(iii) $S_{p}(\varepsilon, a, \omega):=\inf _{u \in M_{p}(\omega) \backslash\{0\}} I_{\varepsilon, a, \omega}(u)$.

(iv) Let $\eta \in C(\mathbb{R})$ be a cut-off function such that

$$
\begin{gathered}
\eta(t):= \begin{cases}1 & \text { if }|t|<R, \\
\frac{R}{t} & \text { if }|t| \geq R,\end{cases} \\
\beta_{R}(u):=\int_{\mathbb{R}^{N}} x \eta(|x|)\left|u^{+}\right|^{p} d x \quad \forall u \in M_{p}\left(\mathbb{R}^{N}\right) .
\end{gathered}
$$

(v) When $a(x) \equiv \alpha>0$, we denote by $\bar{v}_{\varepsilon, \alpha, \omega}$ the minimizer of $S_{p}(\varepsilon, \alpha, \omega)$ which is radially symmetric with respect to the origin, and $\bar{v}_{1, \alpha, \mathbb{R}^{N}}$ is simply denoted by $\bar{v}_{\alpha}$.

(vi) $\varphi_{r} \in C_{0}^{\infty}\left(\mathbb{R}^{N}\right)$ stands for a cut-off function such that $\varphi_{r}$ is radially symmetric with respect to the origin and

$$
\begin{aligned}
\varphi_{r}(x)=1 & \text { if }|x|<\frac{r}{2}, \\
0 \leq \varphi_{r}(x) \leq 1 & \text { if } \frac{r}{2} \leq|x|<r \\
\varphi_{r}(x)=0 & \text { if }|x| \geq r .
\end{aligned}
$$

We also denote $\varphi_{\varepsilon, r}(x):=\varphi_{r}(\varepsilon x)$.

For any $y_{\varepsilon} \in \partial K / \varepsilon$, we put

$$
v_{\varepsilon, \alpha, y_{\varepsilon}}(x):=\frac{\varphi_{\varepsilon, r}\left(x-y_{\varepsilon}\right) \bar{v}_{1, \alpha, \mathbb{R}^{N}}\left(x-y_{\varepsilon}\right)}{\left\|\varphi_{\varepsilon, r} \bar{v}_{1, \alpha, \mathbb{R}^{N}}\right\|_{L^{p}}},
$$

and $\Phi_{\varepsilon, \alpha}\left(y_{\varepsilon}\right):=v_{\varepsilon, \alpha, y_{\varepsilon}}$ for all $y_{\varepsilon} \in \partial K / \varepsilon$. Here $K$ and $r$ are a compact set and a positive constant which appear in the condition $(\mathrm{A})_{K, r, \mathcal{c}, \bar{c}, \delta, \rho}$ in Section 1.

We occasionally suppress the subscript $\alpha$ when no confusion occurs.

(vii) We denote $a_{\varepsilon}(x):=a(\varepsilon x)$.

(viii) Let $X$ be a Banach manifold and $a \in \mathbb{R}$. Then for $I \in C^{1}(X ; \mathbb{R})$, we put

$$
\begin{aligned}
& {[I \leq a]_{X}:=\{u \in X ; I(u) \leq a\},} \\
& {[I=a]_{X}:=\{u \in X ; I(u)=a\},} \\
& \operatorname{Cr}(I ; X):=\left\{u \in X ;(d I)_{u}=0\right\},
\end{aligned}
$$

where $(d I)_{u}$ represents the Fréchet derivative of $I$ at $u \in X$. 


\section{Variational tools and preliminary facts}

3.1. Variational tools. Our main tool relies on the variational approach. We here prepare some terminology frequently used later on.

Definition 3.1 (Palais-Smale condition). Let $M$ be a $C^{1}$ Banach-Finsler manifold and $J \in$ $C^{1}(M ; \mathbb{R})$.

(a) $\left(u_{n}\right) \subset M$ is called a (PS) $)_{c}$-sequence (Palais-Smale sequence at level $c$ ) if

$$
\left\|(d J)_{u_{n}}\right\|_{\left(T_{u_{n}} M\right)^{*}} \longrightarrow 0, \quad J\left(u_{n}\right) \longrightarrow c \quad \text { as } n \longrightarrow \infty
$$

(b) $J$ is said to satisfy the (PS) $)_{c}$-condition if

$(\mathrm{PS})_{c}$ every $(\mathrm{PS})_{c}$-sequence of $J$ contains a strongly convergent subsequence.

(In the above $T_{u} M$ denotes the tangent space of $M$ at $u$.)

Our approach is based on the following fundamental principle.

Fundamental principle in Morse theory. Suppose that $M$ is a Banach-Finsler manifold and $I \in C^{1}(M)$ satisfies the following assumptions:

(1) I satisfies $(\mathrm{PS})_{c}$-condition for all $c \in[a, b]$;

(2) $[I \leq a]_{M}$ and $[I \leq b]_{M}$ have a "difference in topology."

Then there exists a critical value $\bar{c} \in[a, b]$.

In order to compare the topology of sets, various kinds of topological invariants are known. We will here use the notion of the "category" of sets. We use the following notation.

Definition 3.2. Let $M$ be a topological space, and let $A$ and $\bar{x}$ be a closed subset and a point of $M$, respectively.

Denote " $A \simeq\{\bar{x}\}$ by $\eta$ in $M$ " if $\eta \in C([0,1] \times A ; M), \eta(0, x)=x$ for all $x \in A$, and $\eta(1, x)=\bar{x}$ for all $x \in A$.

Definition 3.3 (notion of category). Let $X$ be a topological space and let $M, A$ be two closed subsets of $X$ with $A \subset M$. Then the category of $A$ relative to $M$, denoted by $n=$ $\operatorname{cat}_{M}[A]$, is defined as the smallest number among $m$ such that $\left(A_{j}\right)_{j=1}^{m}$ is a closed contractible covering of $A$ in $M$, that is, there exists a closed covering $\left(A_{j}\right)_{j=1}^{m}$ of $A$ in $M$, $x_{j} \in M$, and $\eta_{j} \in C\left([0,1] \times A_{j} ; M\right)$ such that $A_{j} \simeq\left\{x_{j}\right\}$ by $\eta_{j}$ in $M$ for all $j=1,2, \ldots, m$.

We simply denote cat $\Omega[\Omega]$ by cat $\Omega$.

In terms of this notion, Ljusternik-Schnirelman theorem (category version) reads as follows.

Proposition 3.4 (Ljusternik-Schnirelman theorem, category version [12, Theorem 5.19]). Suppose that $M$ is a $C^{1,1}$ Banach-Finsler manifold, $I \in C^{1}(M)$, and $a=\inf _{M} I>$ $-\infty$. Suppose also that for some $b^{\prime}>b>a, I$ satisfies $(\mathrm{PS})_{c}$ for all $c \in\left[a, b^{\prime}\right]$ and $\operatorname{Cr}(I ; M) \cap$ $[I=b]_{M}=\varnothing$.

Then $[I \leq b]_{M}$ contains at least cat $[I \leq b]_{M}$ critical points. 
In this paper, we use the variational method on the constraint manifold. In order to guarantee that the critical point on the manifold gives the critical point in the original space, we need the following version of Lagrange multiplier rule.

Proposition 3.5 (Lagrange multiplier rule, [12, Proposition 5.12]). Let $X$ be a Banach space, $\psi \in C^{2}(X ; \mathbb{R})$, and $J \in C^{1}(X ; \mathbb{R})$. Let $M=\{u \in X \mid \psi(u)=1\}$. Assume that $(d \psi)_{u} \neq$ 0 in $X^{*}$ for any $u \in M$.

Then $\|(d J)\|_{\left(T_{u} M\right)^{*}}=\min _{C \in \mathbb{R}}\left\|(d J)_{u}-C(d \psi)_{u}\right\|$ holds. In particular, $u \in M$ is a critical point of $J$ restricted in $M$ if and only if there exists $C \in \mathbb{R}$ such that $(d J)_{u}=C(d \psi)_{u}$ in $X^{*}$.

In the proof of Theorem 1.1, we have to compare the category of two sets. For this purpose we use the following comparison theorem of category.

Proposition 3.6 (comparison theorem for category). Let $a$ and $b$ be closed subsets of topological spaces $A$ and $B$, respectively. Suppose that there exist $\Phi \in C(a ; b)$ and $\beta \in C(B ; A)$ such that $\beta \circ \Phi$ is homotopically equivalent to the natural injection from a to $A$. Then, $\operatorname{cat}_{B}[b] \geq \operatorname{cat}_{A}[a]$.

Proof. Let $m=\operatorname{cat}_{B}[b]$. Since $\beta \circ \Phi$ is homotopically equivalent to the injection from $a$ to $A$, there exists $f \in C([0,1] \times a ; A)$ such that for all $x \in a$,

$$
f(0, x)=x, \quad f(1, x)=\beta \circ \Phi(x) .
$$

Since $m=\operatorname{cat}_{B}[b]$, there exist a family of closed subsets $b_{j} \subset B$, a family of mappings $\eta_{j} \in C\left([0,1] \times b_{j} ; B\right)$, and $u_{j} \in B$ for $j=1,2, \ldots, m$ such that

$$
b_{j} \simeq\left\{u_{j}\right\} \text { by } \eta_{j} \text { in } B \quad \forall j=1,2, \ldots, m .
$$

Let $a_{j}=\Phi^{-1}\left(b_{j}\right) \subset a$. Then it is easy to see that $a=\bigcup_{j=1}^{m} a_{j}$ and $a_{j}$ is closed in $A$.

Set

$$
g_{j}(t, x)= \begin{cases}f(2 t, x) & \forall(t, x) \in\left[0, \frac{1}{2}\right] \times a_{j}, \\ \beta \circ \eta_{j}(2 t-1, \Phi(x)) & \forall(t, x) \in\left[\frac{1}{2}, 1\right] \times a_{j} .\end{cases}
$$

Then it is easy to see that

$$
\begin{gathered}
\lim _{t \uparrow 1 / 2} g_{j}(t, x)=f(1, x)=\beta \circ \Phi(x), \\
\lim _{t \downarrow 1 / 2} g_{j}(t, x)=\beta \circ \eta_{j}(0, \Phi(x))=\beta \circ \Phi(x)
\end{gathered}
$$

holds for all $x \in a_{j}$. Hence $g_{j} \in C\left([0,1] \times a_{j} ; A\right)$.

It is also obvious that for all $x \in a_{j}, g_{j}(0, x)=f(0, x)=x$ and $g_{j}(1, x)=\beta \circ \eta_{j}(1$, $\Phi(x))=\beta\left(u_{j}\right) \in A$.

Therefore it holds that $a=\bigcup_{j=1}^{m} a_{j}, a_{j}$ is a closed subset of $A$, and $a_{j} \simeq\left\{\beta\left(u_{j}\right)\right\}$ by $g_{j}$ in $A$. Hence, by the definition of the category cat ${ }_{A}[a]$, we have $m \leq \operatorname{cat}_{A}[a]$.

In order to prove the existence of the critical point which has the higher energy, we use the following version of minimax principle. 
Proposition 3.7 (minimax principle). Let $M$ be a $C^{1,1}$ Banach-Finsler manifold and let $A$ be a metric space. Suppose $A_{0} \subset A$ is a compact subset, $\varphi \in C\left(\partial A_{0} ; M\right)$, and $I \in C^{1}(M ; \mathbb{R})$. Also let $\Gamma=\left\{\gamma \in C\left(A_{0} ; M\right) ;\left.\gamma\right|_{\partial A_{0}}=\varphi\right\} \neq \varnothing$ and let $c=\inf _{\gamma \in \Gamma} \max _{y \in A_{0}} I \circ \gamma(y)>-\infty$.

If I satisfies $(\mathrm{PS})_{c}$ and $\sup _{y \in \partial A_{0}} I \circ \varphi(y)<c$, then $c$ gives a critical value of $I$.

Proof. Suppose the conclusion is false. Then the standard deformation lemma (see, e.g., [11, Theorem II.3.11]) implies that for $\bar{\varepsilon}=\left(c-\sup _{y \in \partial A_{0}} I \circ \varphi(y)\right) / 2$, there exist $\varepsilon \in(0, \bar{\varepsilon})$ and $f \in C([0,1] \times M ; M)$ such that

$$
\begin{gathered}
f\left(1,[I \leq c+\varepsilon]_{M}\right) \subset[I \leq c-\varepsilon]_{M}, \\
f(t, u)=u \quad \forall u \in[I \leq c-\bar{\varepsilon}]_{M} .
\end{gathered}
$$

Take any $\gamma_{\varepsilon} \in \Gamma$ such that $\max _{y \in A_{0}} I \circ \gamma_{\varepsilon}(y)<c+\varepsilon$. Let $\gamma^{\prime}(\cdot)=f\left(1, \gamma_{\varepsilon}(\cdot)\right) \in C\left(A_{0} ; M\right)$. Then by the choice of $\bar{\varepsilon}$, we have $\gamma_{\varepsilon}(y) \in[I \leq c-\bar{\varepsilon}]_{M}$ for all $y \in \partial A_{0}$. Hence, in view of (3.7), it is obvious that for all $y \in \partial A_{0}$,

$$
\gamma^{\prime}(y)=f\left(1, \gamma_{\varepsilon}(y)\right)=\gamma_{\varepsilon}(y)=\varphi(y)
$$

Therefore $\gamma^{\prime} \in \Gamma$ and, in view of (3.6), we have

$$
c=\inf _{\gamma \in \Gamma} \max _{y \in A_{0}} I \circ \gamma(y) \leq \max _{y \in A_{0}} I \circ \gamma^{\prime}(y) \leq c-\varepsilon<c,
$$

a contradiction.

3.2. Preliminary facts. Setting $v(x)=u(\varepsilon x)$, (the weak form of) problem $(\mathrm{P})_{\varepsilon}$ can be rewritten as

$\left(\mathrm{P}^{\prime}\right)_{\varepsilon}$

$$
-\Delta v+a(\varepsilon x) v=|v|^{p-2} v, \quad v \geq 0, v \in H_{0}^{1}\left(\frac{\Omega}{\varepsilon}\right) .
$$

As for $\left(\mathrm{P}^{\prime}\right)_{\mathcal{E}}$, the following fact is well known. For the convenience, we briefly give the sketch of proof.

Proposition 3.8 (variational formulation of $\left.\left(\mathrm{P}^{\prime}\right)_{\varepsilon}\right)$. To find nontrivial solutions of $\left(\mathrm{P}^{\prime}\right)_{\varepsilon}$ is equivalent to

(V) find critical points of $I_{1, a_{\varepsilon}, \Omega / \varepsilon}$ on $M_{p}(\Omega / \varepsilon)$.

Proof. Sufficiency of (V). Assume that (V) has a solution, that is, there exists $u \in M_{p}(\Omega / \varepsilon)$ which is a critical point of $I_{1, a_{\varepsilon}, \Omega / \varepsilon}$. Let $\psi(u)=\int_{\Omega / \varepsilon}\left|u^{+}\right| p$. Since $u \in M_{p}(\Omega / \varepsilon)$, it is obvious that $(d \psi)_{u}(u)=p \int_{\Omega / \varepsilon}\left|u^{+}\right|^{p}=p \neq 0$ and $(d \psi)_{u} \neq 0$ in $\left(H_{0}^{1}(\Omega / \varepsilon)\right)^{*}$. Therefore, by Proposition 3.5, there exists $C \in \mathbb{R}$ such that

$$
\left(d I_{1, a_{\varepsilon}, \Omega / \varepsilon}\right)_{u}(h)=C(d \psi)_{u}(h) \quad \forall h \in H_{0}^{1}\left(\frac{\Omega}{\varepsilon}\right) .
$$


Testing (3.11) with $h=u$, we get

$$
I_{1, a_{\varepsilon}, \Omega / \varepsilon}(u)=\int_{\Omega / \varepsilon}\left(|\nabla u|^{2}+a_{\varepsilon}(x)|u|^{2}\right)=C p \int_{\Omega / \epsilon}\left|u^{+}\right|^{p}=C p
$$

since $u \in M_{p}(\Omega / \varepsilon)$.

Then, by virtue of the fact that $u \neq 0$ and $a_{\varepsilon}>0$, we have

$$
C=\frac{I_{1, a_{\varepsilon}, \Omega / \varepsilon}(u)}{p}>0
$$

Testing also (3.11) with $h=u^{-}=\min (0, u)$, we obtain

$$
\int_{\Omega / \varepsilon}\left(\left|\nabla u^{-}\right|^{2}+a_{\varepsilon}(x)\left|u^{-}\right|^{2}\right)=C p \int_{\Omega / \varepsilon}\left|u^{+}\right|^{p-2} u^{+} u^{-}=0
$$

whence follows $u^{-}=0$ and $u=u^{+} \geq 0$.

Then it is easy to check that $v=\left(I_{1, a_{\varepsilon}, \Omega / \varepsilon}(u) / p\right)^{1 /(p-2)} u$ gives a (nontrivial) solution of $\left(\mathrm{P}^{\prime}\right)_{\varepsilon}$.

Necessity of (V) also follows from arguments similar to those above.

For $S_{p}(\varepsilon, \alpha, \omega)$, it is well known that the following result holds.

Proposition 3.9 (existence and uniqueness for ground state in $\mathbb{R}^{N}$ [9]). For any $\varepsilon>$ 0 and $\alpha>0$, there exists a positive minimizer $\bar{v}_{\varepsilon, \alpha, \mathbb{R}^{N}}$ for $S_{p}\left(\varepsilon, \alpha, \mathbb{R}^{N}\right.$ ) which is unique (up to translation) and radially symmetric with respect to the origin. Especially, the map $\alpha \mapsto$ $S_{p}\left(\varepsilon, \alpha, \mathbb{R}^{N}\right)$ is continuous.

As we will see, the nontriviality of the topology of some level sets of $I_{1, a_{\varepsilon}, \Omega / \varepsilon}$ is the consequence of the nontriviality of that of $\partial K$. In order to discuss this relationship between the level set of $I_{1, a_{\varepsilon}, \Omega / \varepsilon}$ (in function space) and $\partial K / \varepsilon\left(\right.$ in $\mathbb{R}^{N}$ ), we use the "truncated barycenter" $\beta_{R}(u)$ and a family of comparison function $v_{\varepsilon, \alpha, y_{\varepsilon}}$ where $y_{\varepsilon} \in \partial K / \varepsilon$ (see Section 2.2 for definitions).

It is obvious that $\left|\beta_{R}(u)\right| \leq R$ holds for all $u \in M_{p}\left(\mathbb{R}^{N}\right)$. Moreover, if the (intuitive) barycenter of $u \in M_{p}$ is near "infinity", then $\beta_{R}(u)$ is located near $\partial B_{R}=\left\{x \in \mathbb{R}^{N}|| x \mid=\right.$ $R\}$. Namely, the following holds.

LEMma 3.10 (the range of truncated barycenter). (a) For any $\alpha>0, \mid \beta_{R} \circ \Phi_{\varepsilon, \alpha}\left(y_{\varepsilon}\right)-$ $R y_{\varepsilon} /\left|y_{\varepsilon}\right| \mid=o(1)$ as $\varepsilon \rightarrow 0$ uniformly in $y \in \partial K$, where $y_{\varepsilon}=y / \varepsilon$.

(b) Suppose that $u \in M_{p}\left(\mathbb{R}^{N}\right)$ and $\left(y_{n}\right) \subset \mathbb{R}^{N}$ satisfies $\left|y_{n}\right| \rightarrow \infty$ as $n \rightarrow \infty$. Then $\left|\beta_{R}\left(u\left(\cdot-y_{n}\right)\right)\right| \rightarrow R$ as $n \rightarrow \infty$.

Proof. (a) Take any $\alpha>0, \varepsilon>0, y \in \partial K$ and set $y_{\varepsilon}=y / \varepsilon$. Since we will consider the limit $\varepsilon \rightarrow 0$, without loss of generality we can assume that

$$
B\left(y_{\varepsilon}, \frac{\sqrt{\left|y_{\varepsilon}\right|}}{2}\right) \subset B\left(0, \frac{\left|y_{\varepsilon}\right|}{2}\right)^{c} \subset B(0, R)^{c} .
$$


Then it follows that

$$
\begin{aligned}
\left|\beta_{R}\left(v_{\varepsilon, \alpha, y_{\varepsilon}}\right)-R \frac{y_{\varepsilon}}{\left|y_{\varepsilon}\right|}\right| \\
=\left.\left|\int_{\mathbb{R}^{N}} x \eta(|x|)\right| v_{\varepsilon, \alpha, y_{\varepsilon}}(x)\right|^{p} d x-R \frac{y_{\varepsilon}}{\left|y_{\varepsilon}\right|} \int_{\mathbb{R}^{N}}\left|v_{\varepsilon, \alpha, y_{\varepsilon}}(x)\right|^{p} d x \mid \\
\leq\left.\left|\int_{B\left(y_{\varepsilon}, \sqrt{\left|y_{\varepsilon}\right|} / 2\right)} R\left(\frac{x}{|x|}-\frac{y_{\varepsilon}}{\left|y_{\varepsilon}\right|}\right)\right| v_{\varepsilon, \alpha, y_{\varepsilon}}(x)\right|^{p} d x \mid \\
\quad+\left.\left|\int_{B\left(y_{\varepsilon}, \sqrt{\left|y_{\varepsilon}\right|} / 2\right)^{c}}\left(x \eta(|x|)-R \frac{y_{\varepsilon}}{\left|y_{\varepsilon}\right|}\right)\right| v_{\varepsilon, \alpha, y_{\varepsilon}}(x)\right|^{p} d x \mid \\
=(A)+(B) .
\end{aligned}
$$

As for $(A)$, we find that, in view of (3.15),

$$
\begin{aligned}
\left|\frac{x}{|x|}-\frac{y_{\varepsilon}}{\left|y_{\varepsilon}\right|}\right| & \leq \frac{\left|y_{\varepsilon}\right|}{|x|\left|y_{\varepsilon}\right|}\left|x-y_{\varepsilon}\right|+\frac{\left|y_{\varepsilon}\right||| x|-| y_{\varepsilon}||}{|x|\left|y_{\varepsilon}\right|} \\
& \leq 2 \frac{\left|x-y_{\varepsilon}\right|}{|x|} \leq 2 \frac{\sqrt{\left|y_{\varepsilon}\right|} / 2}{\left|y_{\varepsilon}\right| / 2} \\
& =2 \frac{1}{\sqrt{\left|y_{\varepsilon}\right|}}
\end{aligned}
$$

for all $x \in B\left(y_{\varepsilon}, \sqrt{\left|y_{\varepsilon}\right|} / 2\right)$.

Then, since $\left\|\varphi_{\varepsilon, r} \bar{v}_{\alpha}\right\|_{L^{p}\left(\mathbb{R}^{N}\right)} \rightarrow\left\|\bar{v}_{\alpha}\right\|_{L^{p}\left(\mathbb{R}^{N}\right)}$ as $\varepsilon \rightarrow 0$, for suitable positive constant $C_{1}$ and $C_{2}$, we have

$$
\begin{aligned}
(A)+(B) & \leq \frac{\left(\left(2 R / \sqrt{\left|y_{\varepsilon}\right|}\right) \int_{\mathbb{R}^{N}}\left|\bar{v}_{\alpha}\right|^{p}+2 R \int_{B\left(0, \sqrt{\left|y_{\varepsilon}\right|} / 2\right)^{c}}\left|\bar{v}_{\alpha}\right|^{p}\right)}{\|\left.\varphi_{\varepsilon, r} \bar{v}_{\alpha}\right|_{L^{p}\left(\mathbb{R}^{N}\right)} ^{p}} \\
& \leq \frac{\sqrt{\varepsilon}}{\min _{y \in \partial K} \sqrt{|y|}} C_{2}+C_{1} \int_{B\left(0, \min _{y \in \partial K} \sqrt{|y| /(2 \sqrt{\varepsilon}))^{c}}\right.}\left|\bar{v}_{\alpha}\right|^{p} \longrightarrow 0
\end{aligned}
$$

as $\varepsilon \rightarrow 0$, uniformly in $y \in \partial K$ (recall that $\min _{y \in \partial K} \sqrt{|y|}>0$ since $0 \in \operatorname{int} K$ ).

(b) The argument is essentially the same as in [4, proof of Lemma 3.4].

In view of the principle of Morse theory, in order to establish the existence of critical points of $I_{1, a_{\varepsilon}, \Omega / \varepsilon}$, it is enough to verify the existence of a pair of level sets of $I_{1, a_{\varepsilon}, \Omega / \varepsilon}$ which have a difference in topology. The existence of such a pair of level sets of $I_{1, a_{\varepsilon}, \Omega / \varepsilon}$ is the consequence of the existence of that of $I_{1, b_{c, \bar{c}, \rho}, \mathbb{R}^{N}}$, the "limiting functional" associated to $I_{1, a_{\varepsilon}, \Omega / \varepsilon}$ with suitable weight function $b_{c, \bar{c}, \rho}(x)$.

Let $b_{c, \bar{c}, \rho}(x) \in C\left(\mathbb{R}^{N}\right)$ be a function which satisfies the following condition $(\mathrm{B})_{c, \bar{c}, \rho}$ for some positive number $c \in[\underline{c}, \bar{c})$. 
$(\mathrm{B})_{c, \bar{c}, \rho}: b_{c, \bar{c}, \rho}(x)=\chi(|x|)$ where

$$
\chi(t)= \begin{cases}\bar{c} & \text { for } t \leq \frac{\rho}{2} \\ -\frac{2(\bar{c}-c)}{\rho} t+2 \bar{c}-c & \text { for } \frac{\rho}{2}<t \leq \rho, \\ c & \text { for } \rho<t .\end{cases}
$$

Note that by the assumption (ii) of $(\mathrm{A})_{K, r, c, \bar{c}, \delta, \rho}$ and the definition above, for any $\varepsilon \in$ $(0,1)$,

$$
\begin{gathered}
a_{\varepsilon}(x)=a(\varepsilon x) \geq b_{c, \bar{c}, \rho}(x)-(c-\underline{c}) \quad \forall x \in \frac{\Omega}{\varepsilon}, \\
b_{c, \bar{c}, \rho}(x) \geq c \quad \forall x \in \mathbb{R}^{N} .
\end{gathered}
$$

We next investigate the topology of the level set of $I_{1, b_{c, \bar{c}, \rho}, \mathbb{R}^{N}}$ near its infimum level.

3.3. Limiting problem. Hereafter we fix positive constants $c, \bar{c}, \rho$. Let $b_{c, \bar{c}, \rho}(x)$ be the function satisfying $(B)_{c, \bar{c}, \rho}$ in the previous subsection. Throughout this subsection, we denote the limiting functional $I_{1, b_{c, \bar{c}}, \mathbb{R}^{N}}$ as $I_{c, \infty}$.

In view of Proposition 3.9, we have the following.

Lemma 3.11 (inf is not achieved in the limiting problem). (a) $S_{p}\left(1, c, \mathbb{R}^{N}\right)=S_{p}\left(1, b_{c, \bar{c}, \rho}(\cdot)\right.$, $\left.\mathbb{R}^{N}\right)$.

(b) $S_{p}\left(1, b_{c, \bar{c}, \rho}(\cdot), \mathbb{R}^{N}\right)$ is not achieved.

Proof. Suppose that the following claim holds true.

Claim 3.12. Let $b \in L^{N / 2}\left(\mathbb{R}^{N}\right)$ satisfy the following condition for some $c>0$ :

$$
\inf _{x \in \mathbb{R}^{N}} b(x) \geq c, \quad \lim _{|x| \rightarrow \infty} b(x)=c, \quad b(x) \not \equiv c .
$$

Then we have the following:

(a) $S_{p}\left(1, c, \mathbb{R}^{N}\right)=S_{p}\left(1, b(\cdot), \mathbb{R}^{N}\right)$,

(b) $S_{p}\left(1, b(\cdot), \mathbb{R}^{N}\right)$ is not achieved.

Then it is easy to see that Lemma 3.11 follows from the claim above with $b(x)=$ $b_{c, \bar{c}, \rho}(x)$.

Proof of Claim 3.12. (a) It is clear that for all $u \in M_{p}\left(\mathbb{R}^{N}\right)$, we have

$$
\int_{\mathbb{R}^{N}}\left(|\nabla u|^{2}+c|u|^{2}\right) \leq \int_{\mathbb{R}^{N}}\left(|\nabla u|^{2}+b(\cdot)|u|^{2}\right),
$$

whence follows

$$
S_{p}\left(1, c, \mathbb{R}^{N}\right) \leq S_{p}\left(1, b(\cdot), \mathbb{R}^{N}\right) .
$$

To get the converse inequality, we will use some special sequence $\left(v_{n}\right)$. Let $\bar{v}_{c} \in M_{p}\left(\mathbb{R}^{N}\right)$ be a positive minimizer of $S_{p}\left(1, c, \mathbb{R}^{N}\right)$ whose existence is guaranteed by Proposition 3.9. 
Let $\left(y_{n}\right) \subset \mathbb{R}^{N}$ be any sequence which satisfies $\left|y_{n}\right| \rightarrow \infty$ as $n \rightarrow \infty$. Set $v_{n}(\cdot)=\bar{v}_{c}\left(\cdot-y_{n}\right)$. Now we will show that

$$
\left|\int_{\mathbb{R}^{N}}\left(\left|\nabla v_{n}\right|^{2}+b(\cdot)\left|v_{n}\right|^{2}\right)-\int_{\mathbb{R}^{N}}\left(\left|\nabla v_{n}\right|^{2}+c\left|v_{n}\right|^{2}\right)\right| \longrightarrow 0 \quad \text { as } n \longrightarrow \infty .
$$

Take any $\varepsilon>0$. The fact $\lim _{|x| \rightarrow \infty} b(x)=c$ allows us to take $R_{1}$ such that

$$
|b(x)-c|<\frac{\varepsilon}{\left(2\left\|\bar{v}_{c}\right\|_{2}^{2}\right)}
$$

holds for any $|x|>R_{1}$.

Moreover, by virtue of $\bar{v}_{c} \in H^{1}\left(\mathbb{R}^{N}\right) \hookrightarrow L^{2^{*}}\left(\mathbb{R}^{N}\right)$, we can choose $R_{2}$ so large that

$$
\int_{B\left(0, R_{2}\right)^{c}}\left|\bar{v}_{c}(\cdot)\right|^{2^{*}}<\left(\frac{\varepsilon}{\left(2\|b\|_{N / 2}\right)}\right)^{N /(N-2)} .
$$

Since $\left|y_{n}\right| \rightarrow \infty$ as $n \rightarrow \infty$, it is also easy to see that, for $R_{1}$ and $R_{2}$ above,

$$
B\left(y_{n}, R_{2}\right) \subset B\left(0, R_{1}\right)^{c}
$$

holds for large $n$.

Then we have

$$
\begin{aligned}
& \left|\int_{\mathbb{R}^{N}}\left(\left|\nabla v_{n}\right|^{2}+b(\cdot)\left|v_{n}\right|^{2}\right)-\int_{\mathbb{R}^{N}}\left(\left|\nabla v_{n}\right|^{2}+c\left|v_{n}\right|^{2}\right)\right| \\
& \quad \leq\left|\int_{B\left(y_{n}, R_{2}\right)}\right| b(\cdot)-\left.c|| v_{n}\right|^{2}|+| \int_{B\left(y_{n}, R_{2}\right)^{c}}|b(\cdot)-c|\left|v_{n}\right|^{2} \mid \\
& \quad=:(\mathrm{A})+(\mathrm{B}) .
\end{aligned}
$$

Using (3.25) and (3.27), we have

$$
|(\mathrm{A})| \leq \int_{B\left(0, R_{1}\right)^{c}}|b(\cdot)-c|\left|v_{n}\right|^{2} \leq \frac{\varepsilon}{2} .
$$

Moreover (3.26) and the fact that $|b(\cdot)-c|=b(\cdot)-c \leq b(\cdot)$ yield that

$$
\begin{aligned}
|(\mathrm{B})| & \leq \int_{B\left(y_{n}, R_{2}\right)^{c}} b(\cdot)\left|v_{n}(\cdot)\right|^{2} \\
& \leq\left(\int_{B\left(y_{n}, R_{2}\right)^{c}}|b(\cdot)|^{N / 2}\right)^{2 / N}\left(\int_{B\left(0, R_{2}\right)^{c}}\left|\bar{v}_{c}(\cdot)\right|^{2^{*}}\right)^{(N-2) / N} \\
& \leq \frac{\varepsilon}{2}
\end{aligned}
$$

Thus (3.24) follows from (3.28), (3.29), and (3.30).

Combining (3.24) with

$$
\int_{\mathbb{R}^{N}}\left(\left|\nabla v_{n}\right|^{2}+c\left|v_{n}\right|^{2}\right)=\int_{\mathbb{R}^{n}}\left(\left|\nabla \bar{v}_{c}\right|^{2}+c\left|\bar{v}_{c}\right|^{2}\right)=S_{p}\left(1, c, \mathbb{R}^{N}\right),
$$


we have

$$
S_{p}\left(1, c, \mathbb{R}^{N}\right) \geq S_{p}\left(1, b(\cdot), \mathbb{R}^{N}\right) .
$$

(b) As in (a), let $\bar{v}_{c}$ be a positive minimizer for $S_{p}\left(1, c, \mathbb{R}^{N}\right)$. Suppose that the claim is false, that is, there exists $w \in M_{p}\left(\mathbb{R}^{N}\right)$ such that

$$
\int_{\mathbb{R}^{N}}\left(|\nabla w|^{2}+b(\cdot)|w|^{2}\right) d x=S_{p}\left(1, b(\cdot), \mathbb{R}^{N}\right) .
$$

Then, by virtue of $b(x) \geq c$ and $S_{p}\left(1, c, \mathbb{R}^{N}\right)=S_{p}\left(1, b(\cdot), \mathbb{R}^{N}\right)$, we get

$$
\begin{aligned}
S_{p}\left(1, c, \mathbb{R}^{N}\right) & \leq \int_{\mathbb{R}^{N}}\left(|\nabla w|^{2}+c|w|^{2}\right) \\
& \leq \int_{\mathbb{R}^{N}}\left(|\nabla w|^{2}+b(\cdot)|w|^{2}\right) \\
& =S_{p}\left(1, b(\cdot), \mathbb{R}^{N}\right) \\
& =S_{p}\left(1, c, \mathbb{R}^{N}\right),
\end{aligned}
$$

that is, $S_{p}\left(1, c, \mathbb{R}^{N}\right)=\int_{\mathbb{R}^{N}}\left(|\nabla w|^{2}+c|w|^{2}\right)$. Then from Proposition 3.9, we find that $w>0$ in $\mathbb{R}^{N}$. Therefore, in view of $b(x) \geq c$ and $b(x) \not \equiv c$, we have

$$
\begin{aligned}
S_{p}\left(1, c, \mathbb{R}^{N}\right) & =\int_{\mathbb{R}^{N}}\left(|\nabla w|^{2}+c|w|^{2}\right) \\
& <\int_{\mathbb{R}^{N}}\left(|\nabla w|^{2}+b(\cdot)|w|^{2}\right) \\
& =S_{p}\left(1, b(\cdot), \mathbb{R}^{N}\right) \\
& =S_{p}\left(1, c, \mathbb{R}^{N}\right),
\end{aligned}
$$

which leads to a contradiction.

This result implies that all the minimizing sequences possess no convergent subsequence. Combining this fact with the compactness of embedding $H^{1} \hookrightarrow L_{\text {loc }}^{p}$, we find the following.

LEMMA 3.13 (behavior of minimizing sequences of the limiting problem [4, Lemma 2.2]). For any minimizing sequence $\left(v_{n}\right) \subset M_{p}\left(\mathbb{R}^{N}\right)$ of $I_{c, \infty}$, there exists $\left(y_{n}\right) \subset \mathbb{R}^{N}$ such that $\left|y_{n}\right| \rightarrow$ $\infty$ and $v_{n}(\cdot)=\bar{v}_{c}\left(\cdot-y_{n}\right)+o(1)$ in $H^{1}\left(\mathbb{R}^{N}\right)$ as $n \rightarrow \infty$, where $\bar{v}_{c}(x)=\bar{v}_{1, c, \mathbb{R}^{N}}(x)$ is a (unique) minimizer of $S_{p}\left(1, c, \mathbb{R}^{N}\right)$.

Lemma 3.13 says that, for any $v \in M_{p}\left(\mathbb{R}^{N}\right)$ such that $I_{c, \infty}(v)$ is very close to its infimum $S_{p}\left(1, b_{c, \bar{c}, \rho}(\cdot), \mathbb{R}^{N}\right), v$ is almost concentrated at infinity. So by Lemma $3.10(\mathrm{~b})$, we find that $\left|\beta_{R}(v)\right| \simeq R$. Thus we get the following first key result stating the nontriviality of some level set of $I_{c, \infty}$ near its minimum level.

Proposition 3.14 (concentration lemma at infinity for the limiting functional). For all $s \in(0, R)$ and positive numbers $\rho, \underline{c}, \bar{c}$ with $\underline{c}<\bar{c}$, there exists $\alpha>0$ which satisfies the following. If $v \in M_{p}\left(\mathbb{R}^{N}\right)$ satisfies $I_{c, \infty}(v) \leq S_{p}\left(1, b_{c, \bar{c}, \rho}(\cdot), \mathbb{R}^{N}\right)+\alpha$ for $b_{c, \bar{c}, \rho}(x)$ satisfying the condition $(\mathrm{B})_{c, \bar{c}, \rho}$ with some $c \in[\underline{c},(\underline{c}+\bar{c}) / 2]$, then $\beta_{R}(v) \notin B(0, s)$ holds. 
Proof. Suppose that the claim is not true. Then we find that for some $\rho>0, s \in(0, R)$, $\underline{c}, \bar{c}$ with $\underline{c}<\bar{c}$, there exist $\left(c_{n}\right) \subset[\underline{c},(\underline{c}+\bar{c}) / 2],\left(b_{c_{n}, \bar{c}, \rho}(x)\right) \subset C\left(\mathbb{R}^{N}\right)$ defined by $(\mathrm{B})_{c_{n}, \bar{c}, \rho}$, $\left(v_{n}\right) \subset M_{p}\left(\mathbb{R}^{N}\right)$ such that

$$
\begin{gathered}
I_{c_{n}, \infty}\left(v_{n}\right)=S_{p}\left(1, b_{c_{n}, \bar{c}, \rho}(\cdot), \mathbb{R}^{N}\right)+o(1), \\
\beta_{R}\left(v_{n}\right) \in B(0, s)
\end{gathered}
$$

as $n \rightarrow \infty$, passing to subsequence if necessary.

Since $\left(c_{n}\right) \subset[\underline{c},(\underline{c}+\bar{c}) / 2]$, taking further subsequence if necessary, there exists $c \in$ $[\underline{c},(\underline{c}+\bar{c}) / 2]$ such that

$$
c_{n}=c+o(1)
$$

Let $b_{c, \bar{c}, \rho}(x) \in C(\Omega)$ be a function defined by $(\mathrm{B})_{c, \bar{c}, \rho}$.

Then, by virtue of the condition $(\mathrm{B})_{\mathcal{c}_{n}, \bar{c}, \rho}$ and (3.38), we have

$$
\left|b_{c_{n}, \bar{c}, \rho}(x)-b_{c, \bar{c}, \rho}(x)\right| \leq\left|c_{n}-c\right|=o(1) \quad \text { uniformly in } x \in \mathbb{R}^{N} .
$$

Also it is easy to see that (3.36) yields the boundedness of $\left(v_{n}\right) \subset H^{1}\left(\mathbb{R}^{N}\right)$. Therefore, it follows that

$$
\left|I_{c_{n}, \infty}\left(v_{n}\right)-I_{c, \infty}\left(v_{n}\right)\right| \leq \int_{\mathbb{R}^{N}}\left|b_{c_{n}, \bar{c}, \rho}(\cdot)-b_{c, \bar{c}, \rho}(\cdot)\right|\left|v_{n}\right|^{2}=o(1)
$$

as $n \rightarrow \infty$.

Furthermore, by Proposition 3.9 and (3.38), it holds that

$$
S_{p}\left(1, c_{n}, \mathbb{R}^{N}\right)=S_{p}\left(1, c, \mathbb{R}^{N}\right)+o(1)
$$

as $n \rightarrow \infty$.

Therefore, by virtue of (3.36), (3.40), and (3.41), we find that $\left(v_{n}\right) \subset M_{p}\left(\mathbb{R}^{N}\right)$ is a minimizing sequence of $I_{c, \infty}$. Hence, together with Lemma 3.13, we have

$$
v_{n}(\cdot)=\bar{v}_{1, c, \mathbb{R}^{N}}\left(\cdot-y_{n}\right)+o(1) \quad \text { in } H^{1}\left(\mathbb{R}^{N}\right)
$$

for some $\left(y_{n}\right) \subset \mathbb{R}^{N}$ with $\left|y_{n}\right| \rightarrow \infty$ as $n \rightarrow \infty$. Consequently, Lemma 3.10(b) combining with the continuity of $\beta_{R}$ implies that

$$
\left|\beta_{R}\left(v_{n}\right)\right|=\left|\beta_{R}\left(\bar{v}_{1, c, \mathbb{R}^{N}}\left(\cdot-y_{n}\right)\right)\right|+o(1)=R+o(1)
$$

as $n \rightarrow \infty$. But this is impossible in view of (3.37).

This proposition says that $\left[I_{c, \infty} \leq S_{p}+\alpha\right]\left(\subset M_{p}\left(\mathbb{R}^{N}\right)\right)$, the infinite-dimensional object, can be compared with the $B(0, s)^{c}\left(\subset \mathbb{R}^{N}\right)$, the finite-dimensional object, with the aid of $\beta_{R}(v)$. We will see in the next subsection that this correspondence between the finitedimensional object and the infinite-dimensional object is also observed in the "original" problem. 
3.4. Original problem. Now we proceed to the analysis of the original problem. Throughout this subsection, we assume that $a(x)$ is a function satisfying the condition $(\mathrm{A})_{K, r, \mathcal{c}, \bar{c}, \delta, \rho}$ for some positive numbers $\rho, \underline{c}, \bar{c}, \delta$ with $\underline{c}<\bar{c}$ and $\delta \in(0, \bar{c}-\underline{c})$. We also assume that $b_{\underline{c}+\delta, \bar{c}}(x)$ is a function defined by the condition $(\mathrm{B})_{\underline{c}+\delta, \bar{c}, \rho}$ in the last part of Section 3.2.

As in the previous subsection, we denote the limiting functional $I_{1, b_{c, \bar{c}}, \mathbb{R}^{N}}$ as $I_{c, \infty}$ and the original functional $I_{1, a_{\varepsilon}, \Omega / \varepsilon}$ as $I_{\mathcal{\varepsilon}}$.

We regard the original functional $I_{\mathcal{\varepsilon}}(v)=\int_{\Omega / \varepsilon}|\nabla v|^{2}+a(\varepsilon x)|v|^{2}$ as a perturbed functional of the limiting functional $I_{\underline{c}+\delta, \infty}$ for suitable $\delta>0$. Furthermore with the aid of $\Phi_{\varepsilon, \underline{c}+\delta}$ (see Section 2.2 for definition), we can embed the topology of $\partial K / \varepsilon$ into the level set of $I_{\varepsilon}$ near its infimum level when $\varepsilon$ is small enough.

Proposition 3.15 (construction of an embedding mapping from $\mathbb{R}^{N}$ to the function space). For any $\delta>0, I_{\varepsilon} \circ \Phi_{\varepsilon, \underline{c}+\delta}\left(y_{\varepsilon}\right) \leq S_{p}\left(1, b_{\underline{c}+\delta, \bar{c}, \rho}(\cdot), \mathbb{R}^{N}\right)+o(1)$ as $\varepsilon \rightarrow 0$ uniformly in $y \in \partial K$ where $y_{\varepsilon}=y / \varepsilon$.

Proof. First we are going to show the following fact.

Claim 3.16. Let $\alpha>0$. Then

$$
\lim _{\varepsilon \rightarrow 0} \int_{\mathbb{R}^{N}}\left(\left|\nabla v_{\varepsilon, \alpha, y_{\varepsilon}}\right|^{2}+\alpha\left|v_{\varepsilon, \alpha, y_{\varepsilon}}\right|^{2}\right)=S_{p}\left(1, \alpha, \mathbb{R}^{N}\right)
$$

uniformly in $y \in \mathbb{R}^{N}$ (see Section 2.2 for the definition of $v_{\varepsilon, \alpha, y_{\varepsilon}}$ ).

Proof of Claim 3.16. In view of

$$
\begin{gathered}
\int_{\mathbb{R}^{N}}\left(\left|\nabla v_{\varepsilon, \alpha, y_{\varepsilon}}\right|^{2}+\alpha\left|v_{\varepsilon, \alpha, y_{\varepsilon}}\right|^{2}\right)=\int_{\mathbb{R}^{N}}\left(\left|\nabla v_{\varepsilon, \alpha, 0}\right|^{2}+\alpha\left|v_{\varepsilon, \alpha, 0}\right|^{2}\right), \\
S_{p}\left(1, \alpha, \mathbb{R}^{N}\right)=\int_{\mathbb{R}^{N}}\left(\left|\nabla \bar{v}_{\alpha}\right|^{2}+\alpha\left|\bar{v}_{\alpha}\right|^{2}\right),
\end{gathered}
$$

we have only to verify that

$$
\lim _{\varepsilon \rightarrow 0} \int_{\mathbb{R}^{N}}\left(\left|\nabla v_{\varepsilon, \alpha, 0}\right|^{2}+\alpha\left|v_{\varepsilon, \alpha, 0}\right|^{2}\right)=\int_{\mathbb{R}^{N}}\left(\left|\nabla \bar{v}_{\alpha}\right|^{2}+\alpha\left|\bar{v}_{\alpha}\right|^{2}\right) .
$$

Note that

$$
\begin{aligned}
& \left|\int_{\mathbb{R}^{N}}\left(\left|\nabla v_{\varepsilon, \alpha, 0}\right|^{2}+\alpha\left|v_{\varepsilon, \alpha, 0}\right|^{2}\right)-\int_{\mathbb{R}^{N}}\left(\left|\nabla \bar{v}_{\alpha}\right|^{2}+\alpha\left|\bar{v}_{\alpha}\right|^{2}\right)\right| \\
& \quad=\left|\frac{\int_{\mathbb{R}^{N}}\left(\left|\nabla\left(\varphi_{\varepsilon, r} \bar{v}_{\alpha}\right)\right|^{2}+\alpha\left|\varphi_{\varepsilon, r} \bar{v}_{\alpha}\right|^{2}\right)}{\left(\int_{\mathbb{R}^{N}}\left|\varphi_{\varepsilon, r} \bar{v}_{\alpha}\right|^{p}\right)^{2 / p}}-\int_{\mathbb{R}^{N}}\left(\left|\nabla \bar{v}_{\alpha}\right|^{2}+\alpha\left|\bar{v}_{\alpha}\right|^{2}\right)\right| .
\end{aligned}
$$

Here it is easy to see that

$$
\left.\left|\int_{\mathbb{R}^{N}}\right| \varphi_{\varepsilon, r} \bar{v}_{\alpha}\right|^{p}-1|=| \int_{\mathbb{R}^{N}}\left|\varphi_{\varepsilon, r} \bar{v}_{\alpha}\right|^{p}-\left.\int_{\mathbb{R}^{N}}\left|\bar{v}_{\alpha}\right|^{p}\left|\leq C \int_{B(0, r / 2 \varepsilon)^{c}}\right| \bar{v}_{\alpha}\right|^{p} \longrightarrow 0
$$

as $\varepsilon \rightarrow 0$. 
Since $\left|\nabla \varphi_{\varepsilon, r}\right|=\varepsilon\left|\nabla \varphi_{r}\right| \leq \varepsilon C_{3}$, we also find that

$$
\begin{aligned}
\mid \int_{\mathbb{R}^{N}} & \left(\left|\nabla\left(\varphi_{\varepsilon, r} \bar{v}_{\alpha}\right)\right|^{2}+\alpha\left|\varphi_{\varepsilon, r} \bar{v}_{\alpha}\right|^{2}\right)-\int_{\mathbb{R}^{N}}\left(\left|\nabla \bar{v}_{\alpha}\right|^{2}+\alpha\left|\bar{v}_{\alpha}\right|^{2}\right) \mid \\
\leq & \left.\int_{B(0, r / 2 \varepsilon)^{c}}\left|\nabla \bar{v}_{\alpha}\right|^{2}|| \varphi_{\varepsilon, r}\right|^{2}-\left.\left.1\left|+\alpha \int_{B(0, r / 2 \varepsilon)^{c}}\right| \bar{v}_{\alpha}\right|^{2}|| \varphi_{\varepsilon, r}\right|^{2}-1 \mid \\
& +2 \int_{B(0, r / 2 \varepsilon)^{c}}\left|\nabla \varphi_{\varepsilon, r}\right|\left|\nabla \bar{v}_{\alpha}\right|\left|\varphi_{\varepsilon, r}\right|\left|\bar{v}_{\alpha}\right|+\int_{B(0, r / 2 \varepsilon)^{c}}\left|\nabla \varphi_{\varepsilon, r}\right|^{2}\left|\bar{v}_{\alpha}\right|^{2} \\
\leq & C_{1} \int_{B(0, r / 2 \varepsilon)^{c}}\left(\left|\nabla \bar{v}_{\alpha}\right|^{2}+\alpha\left|\bar{v}_{\alpha}\right|^{2}\right)+\varepsilon C_{2}\left(\int_{B(0, r / 2 \varepsilon)^{c}}\left|\nabla \bar{v}_{\alpha}\right|^{2}\right)^{1 / 2} \\
& \times\left(\int_{B(0, r / 2 \varepsilon)^{c}}\left|\bar{v}_{\alpha}\right|^{2}\right)^{1 / 2}+\varepsilon^{2} C_{3}^{2} \int_{B(0, r / 2 \varepsilon)^{c}}\left|\bar{v}_{\alpha}\right|^{2} \longrightarrow 0
\end{aligned}
$$

as $\mathcal{\varepsilon} \rightarrow 0$.

Thus (3.47), (3.48), and (3.49) imply the claim.

Note that for all $y \in \partial K$,

$$
\operatorname{supp} v_{\varepsilon, \underline{c}+\delta, y_{\varepsilon}}=B\left(y_{\varepsilon}, \frac{r}{\varepsilon}\right) \subset\left(\frac{\partial K}{\varepsilon}\right)_{r / \varepsilon}=\left\{x \in \mathbb{R}^{N} \mid d\left(x, \frac{\partial K}{\varepsilon}\right) \leq \frac{r}{\varepsilon}\right\} .
$$

Here (i) of $(\mathrm{A})_{K, r, \underline{c}, \bar{c}, \delta, \rho}$ assures that $(\partial K / \varepsilon)_{r / \varepsilon} \subset \Omega / \varepsilon$. Hence we find that $v_{\varepsilon, \underline{c}+\delta, y_{\varepsilon}}=$ $\Phi_{\varepsilon, c+\delta}\left(y_{\varepsilon}\right) \in M_{p}(\Omega / \varepsilon)$.

Also note that by virtue of (iv) of $(\mathrm{A})_{K, r, \mathcal{G}, \bar{c}, \delta, \rho}$, we have

$$
\sup _{(\partial K / \varepsilon)_{r / \varepsilon}} a_{\varepsilon}(x)=\sup _{(\partial K)_{r}} a(x) \leq \underline{c}+\delta .
$$

Then, in view of Claim 3.16 and Lemma 3.11(a), we obtain

$$
\begin{aligned}
I_{\varepsilon} \circ \Phi_{\varepsilon, \underline{c}+\delta}\left(y_{\varepsilon}\right) & =\int_{\Omega / \varepsilon}\left(\left|\nabla v_{\varepsilon, \underline{c}+\delta, y_{\varepsilon}}\right|^{2}+a_{\varepsilon}(\cdot)\left|v_{\varepsilon, \underline{c}+\delta, y_{\varepsilon}}\right|^{2}\right) \\
& \leq \int_{B\left(y_{\varepsilon}, r / \varepsilon\right)}\left(\left|\nabla v_{\varepsilon, \underline{c}+\delta, y_{\varepsilon}}\right|^{2}+\left\{\sup _{(\partial K / \varepsilon)_{r / \varepsilon}} a_{\varepsilon}(\cdot)\right\}\left|v_{\varepsilon, \underline{c}+\delta, y_{\varepsilon}}\right|^{2}\right) \\
& \leq \int_{B\left(y_{\varepsilon}, r / \varepsilon\right)}\left(\left|\nabla v_{\varepsilon, \underline{c}+\delta, y_{\varepsilon}}\right|^{2}+(\underline{c}+\delta)\left|v_{\varepsilon, \underline{c}+\delta, y_{\varepsilon}}\right|^{2}\right) \\
& \longrightarrow S_{p}\left(1, \underline{c}+\delta, \mathbb{R}^{N}\right)=S_{p}\left(1, b_{\underline{c}+\delta, \bar{c}, \rho}(x), \mathbb{R}^{N}\right)
\end{aligned}
$$

as $\varepsilon \rightarrow 0$ uniformly in $y \in \partial K$.

We next prove that under the condition $(\mathrm{A})_{K, r, \mathcal{G}, \bar{c}, \delta, \rho}$, the relation between the level set of functional $I_{c, \infty}$ and $B(0, s)^{c}$ described in Proposition 3.14 still holds for the perturbed functional $I_{\varepsilon}$.

Proposition 3.17 (concentration lemma at infinity for original functional). For any positive numbers $\rho, s, \underline{c}$, and $\bar{c}$ with $\underline{c}<\bar{c}$, there exist $\delta>0, \eta>0$ such that for any $a(x)$ satisfying $(\mathrm{A})_{K, r, \mathcal{G}, \bar{c}, \delta, \rho}$ with some $r>0$ and some closed subset $K$ of $\Omega$, the following holds: there exists 
$\bar{\varepsilon} \in(0,1)$ such that for all $\varepsilon \in(0, \bar{\varepsilon}]$,

$$
\left[I_{\varepsilon} \leq S_{p}\left(1, \underline{c}+\delta, \mathbb{R}^{N}\right)+\eta\right]_{M_{p}(\Omega / \varepsilon)} \neq \varnothing
$$

and if $v \in M_{p}(\Omega / \varepsilon)$ satisfies $I_{\mathcal{\varepsilon}}(v) \leq S_{p}\left(1, \underline{c}+\delta, \mathbb{R}^{N}\right)+\eta$, then $\beta_{R}(v) \notin B(0, s)$ holds.

Proof. Take any positive numbers $\rho, s<R, \underline{c}, \bar{c}$ with $\underline{c}<\bar{c}$.

Then by Proposition 3.14, there exists $\alpha$ such that for all $c \in[\underline{c},(\underline{c}+\bar{c}) / 2], b_{c, \bar{c}, \rho}(x)$ satisfying the condition $(\mathrm{B})_{c, \bar{c}, \rho}$ and $v \in M_{p}\left(\mathbb{R}^{N}\right)$,

$$
I_{c, \infty}(v) \leq S_{p}\left(1, b_{c, \bar{c}, \rho}(\cdot), \mathbb{R}^{N}\right)+\alpha \Longrightarrow \beta_{R}(v) \notin B(0, s) .
$$

Let

$$
\eta=\frac{\alpha}{2}, \quad \delta=\min \left(\frac{\underline{c} \eta}{S_{p}\left(1, \bar{c}, \mathbb{R}^{N}\right)+\eta}, \frac{\bar{c}-\underline{c}}{2}\right) .
$$

Take any function $a(x)$ which satisfies the condition $(\mathrm{A})_{K, r, \underline{c}, \bar{c}, \delta, \rho}$ for some $r>0$.

Then by Proposition 3.15, for $\delta$ above, we see that there exists $\varepsilon^{\prime}>0$ such that for all $\varepsilon \in\left(0, \varepsilon^{\prime}\right]$,

$$
I_{\varepsilon} \circ \Phi_{\varepsilon, \underline{c}+\delta}\left(y_{\varepsilon}\right) \leq S_{p}\left(1, b_{\underline{c}+\delta, \bar{c}, \rho}(\cdot), \mathbb{R}^{N}\right)+\eta
$$

for all $y \in \partial K$.

Hence by Lemma 3.11(a), $\left[I_{\varepsilon} \leq S_{p}\left(1, \underline{c}+\delta, \mathbb{R}^{N}\right)+\eta\right]_{M_{p}(\Omega / \varepsilon)} \neq \varnothing$ for such $\varepsilon$.

Set $\bar{\varepsilon}=\min \left(\varepsilon^{\prime}, 1\right)$ and take any $\varepsilon \in(0, \bar{\varepsilon}], v \in M_{p}(\Omega / \varepsilon)$ such that

$$
I_{\mathcal{\varepsilon}}(v) \leq S_{p}\left(1, b_{\underline{c}+\delta, \bar{c}, \rho}(\cdot), \mathbb{R}^{N}\right)+\eta
$$

Then, by virtue of the condition (ii) of $(\mathrm{A})_{K, r, \underline{c}, \bar{c}, \delta, \rho}$, we find that

$$
\underline{c} \int_{\mathbb{R}^{N}}|v|^{2} \leq \int_{\mathbb{R}^{N}} a_{\varepsilon}(\cdot)|v|^{2} \leq I_{\mathcal{\varepsilon}}(v)
$$

whence follows

$$
\int_{\mathbb{R}^{N}}|v|^{2} \leq \frac{I_{\mathcal{\varepsilon}}(v)}{\underline{c}}
$$

Also note that, in view of (3.20) and $\varepsilon<1$, we have

$$
b_{\underline{c}+\delta, \bar{c}, \rho}(x)-a_{\varepsilon}(x) \leq \delta \quad \forall x \in \frac{\Omega}{\varepsilon} .
$$


Hence, from (3.59) and (3.60), we deduce that

$$
\begin{aligned}
I_{\underline{\underline{c}}+\delta, \infty}(v) & =\int_{\mathbb{R}^{N}}\left(|\nabla v|^{2}+a_{\mathcal{\varepsilon}}(\cdot)|v|^{2}\right)+\int_{\mathbb{R}^{N}}\left(b_{\underline{\underline{c}}+\delta, \bar{c}, \rho}(\cdot)-a_{\mathcal{\varepsilon}}(\cdot)\right)|v|^{2} \\
& \leq I_{\mathcal{\varepsilon}}(v)+\delta \int|v|^{2} \\
& \leq I_{\mathcal{\varepsilon}}(v)+\frac{\delta I_{\mathcal{\varepsilon}}(v)}{\underline{c}} \\
& \leq\left(S_{p}\left(1, b_{\underline{c}+\delta, \bar{c}, \rho}(\cdot), \mathbb{R}^{N}\right)+\eta\right)+\frac{\underline{c} \eta}{S_{p}\left(1, \bar{c}, \mathbb{R}^{N}\right)+\eta} \frac{S_{p}\left(1, b_{\underline{c}+\delta, \bar{c}, \rho}(x), \mathbb{R}^{N}\right)+\eta}{\underline{c}} \\
& \leq S_{p}\left(1, b_{\underline{c}+\delta, \bar{c}, \rho}(\cdot), \mathbb{R}^{N}\right)+\alpha .
\end{aligned}
$$

Therefore (3.54) with $c=\underline{c}+\delta$ yields $\beta_{R}(v) \notin B(0, s)$ (note that by the definition of $\delta$, we have $c=\underline{c}+\delta \in[\underline{c},(\underline{c}+\bar{c}) / 2])$.

Thus we have the conclusion.

\section{Proof of Theorem 1.1}

Now we are in the position to give a proof of Theorem 1.1. In this section, we denote the original functional $I_{1, a_{\varepsilon}, \Omega / \epsilon}$ by $I_{\varepsilon}$. Take any positive numbers $\rho, \underline{c}, \bar{c}$ with $\underline{c}<\bar{c}$ and $R$, $s$ with $R>s$. Then Proposition 3.17 implies that there exist $\delta>0, \eta>0$ such that for any $a(x)$ satisfying $(\mathrm{A})_{K, r, \underline{c}, \bar{c}, \delta, \rho}$ with some $r>0$ and some closed subset $K$ of $\Omega$, the following holds: there exists $\varepsilon_{1} \in(0,1)$ such that for all $\varepsilon \in\left(0, \varepsilon_{1}\right]$,

$$
\begin{gathered}
{\left[I_{\varepsilon} \leq S_{p}\left(1, \underline{c}+\delta, \mathbb{R}^{N}\right)+\eta\right]_{M_{p}(\Omega / \varepsilon)} \neq \varnothing,} \\
\beta_{R}\left(\left[I_{\varepsilon} \leq S_{p}\left(1, \underline{c}+\delta, \mathbb{R}^{N}\right)+\eta\right]_{M_{p}(\Omega / \varepsilon)}\right) \subset B(0, s)^{c} .
\end{gathered}
$$

Take any $a(x)$ which satisfies $(\mathrm{A})_{K, r, \mathcal{c}, \bar{c}, \delta, \rho}$.

Then, by Proposition 3.15 (with $\delta, \eta$ above) and Lemma 3.11(a), we see that there exists $\varepsilon_{2}>0$ such that for all $\varepsilon \in\left(0, \varepsilon_{2}\right]$,

$$
I_{\varepsilon} \circ \Phi_{\varepsilon, \underline{c}+\delta}\left(y_{\varepsilon}\right) \leq S_{p}\left(1, \underline{c}+\delta, \mathbb{R}^{N}\right)+\frac{\eta}{4}
$$

for all $y_{\varepsilon} \in \partial K / \varepsilon$.

Note that since $s<R$, we can take $\rho_{0}$ so small that

$$
(\partial B(0, R))_{\rho_{0}}:=\left\{x \in \mathbb{R}^{N} ; \operatorname{dist}(\partial B(0, R), x) \leq \rho_{0}\right\} \subset B(0, s)^{c} .
$$

Then, by Lemma 3.10(a), we find that there exists $\varepsilon_{3}>0$ such that

$$
\beta_{R} \circ \Phi_{\varepsilon, \underline{c}+\delta}\left(y_{\varepsilon}\right) \in B\left(\frac{y_{\varepsilon}}{\left|y_{\varepsilon}\right|} R, \rho_{0}\right)
$$

for all $\varepsilon \in\left(0, \varepsilon_{3}\right]$ and $y_{\varepsilon} \in \partial K / \varepsilon$. 
It is also obvious that we can take $\varepsilon_{4}>0$ such that for all $\varepsilon \in\left(0, \varepsilon_{4}\right]$,

$$
\partial K / \varepsilon \subset B(0, R)^{c} .
$$

Let $\bar{\varepsilon}=\min \left(\varepsilon_{1}, \varepsilon_{2}, \varepsilon_{3}, \varepsilon_{4}\right)$ and take any $\varepsilon \in(0, \bar{\varepsilon})$.

We are going to verify the following claim which assures the assertion of Theorem 1.1. Claim 4.1. There exist $c_{1}, c_{2}$ such that

(a) $c_{1}$ is not a critical value of $I_{\varepsilon}$ and

$$
m_{1} \geq \operatorname{cat}\left[S^{N-1}\right]=2,
$$

where $m_{1}$ is the number of critical points in $\left[I_{\varepsilon} \leq c_{1}\right]_{M_{p}(\Omega / \varepsilon)}$,

(b) $c_{2}$ is a critical value of $I_{\varepsilon}$ and $c_{1}<c_{2}$.

Proof of Claim 4.1. (a) Without loss of generality, we can assume that there exists $c_{1}$ which satisfies

$$
c_{1} \in\left[S_{p}\left(1, \underline{c}+\delta, \mathbb{R}^{N}\right)+\frac{\eta}{4}, S_{p}\left(1, \underline{c}+\delta, \mathbb{R}^{N}\right)+\frac{2 \eta}{4}\right]
$$

and $c_{1}$ is not a critical value of $I_{\mathcal{E}}$, otherwise we already have infinitely many critical values and the proof is finished.

Then, by (4.3),

$$
\Phi_{\varepsilon, \underline{c}+\delta}\left(\frac{\partial K}{\varepsilon}\right) \subset\left[I_{\varepsilon} \leq S_{p}\left(1, \underline{c}+\delta, \mathbb{R}^{N}\right)+\frac{\eta}{4}\right]_{M_{p}(\Omega / \varepsilon)} \subset\left[I_{\varepsilon} \leq c_{1}\right]_{M_{p}(\Omega / \varepsilon)} .
$$

Let

$$
g\left(t, y_{\varepsilon}\right)= \begin{cases}(1-2 t) \beta_{R} \circ \Phi_{\varepsilon, \underline{c}+\delta}\left(y_{\varepsilon}\right)+\frac{2 t R y_{\varepsilon}}{\left|y_{\varepsilon}\right|} & \forall\left(t, y_{\varepsilon}\right) \in\left[0, \frac{1}{2}\right] \times \frac{\partial K}{\varepsilon}, \\ \frac{2(1-t) R y_{\varepsilon}}{\left|y_{\varepsilon}\right|}+(2 t-1) y_{\varepsilon} & \forall\left(t, y_{\varepsilon}\right) \in\left[\frac{1}{2}, 1\right] \times \frac{\partial K}{\varepsilon} .\end{cases}
$$

Then it is easy to see that

$$
g \in C\left([0,1] \times \frac{\partial K}{\varepsilon} ; \mathbb{R}^{N}\right), \quad g\left(0, y_{\varepsilon}\right)=\beta_{R} \circ \Phi_{\varepsilon, \underline{c}+\delta}\left(y_{\varepsilon}\right), \quad g\left(1, y_{\varepsilon}\right)=y_{\varepsilon} .
$$

Moreover, because of (4.4), (4.5), and (4.6), we have $g\left([0,1], y_{\varepsilon}\right) \subset B(0, s)^{c}$. Hence $g$ is a homotopy between $\beta_{R} \circ \Phi_{\varepsilon, \underline{c}+\delta}$ and the natural injection from $\partial K / \varepsilon$ into $B(0, s)^{c}$, that is,

(I) $\beta_{R} \circ \Phi_{\varepsilon, \underline{c}+\delta}$ is homotopically equivalent to the natural injection from $\partial K / \varepsilon$ into $B(0, s)^{c}$.

Let $A=B(0, s)^{c}, a=\partial K / \varepsilon, B=b=\left[I_{\varepsilon} \leq c_{1}\right], \Phi=\Phi_{\varepsilon, \underline{c}+\delta}$, and $\beta=\beta_{R}$. Then (4.2), (4.9), and (I) assure that all the hypothesis of Proposition 3.6 is fulfilled. Hence

$$
\operatorname{cat}\left[I_{\varepsilon} \leq c_{1}\right] \geq \operatorname{cat}_{B(0, s)^{c}}\left[\frac{\partial K}{\varepsilon}\right]=\operatorname{cat}\left[S^{N-1}\right]=2 .
$$


It is standard to check that $I_{\varepsilon}$ satisfies the (PS)-condition in $M_{p}$. Hence Proposition 3.4 together with (4.12) implies that (4.7) holds.

(b) We will rely on the minimax principle, see Proposition 3.7.

Let $\Gamma=\left\{\gamma \in C\left(K / \varepsilon ; M_{p}\right) ;\left.\gamma\right|_{\partial K / \varepsilon}=\Phi_{\varepsilon, \underline{c}+\delta}\right\}$ and $c_{2}=\inf _{\gamma \in \Gamma} \max _{y \in K / \varepsilon} I_{\varepsilon} \circ \gamma$. Since obviously $\Phi_{\varepsilon, \underline{c}+\delta}$ can be extended to $K / \varepsilon$ and $I_{\mathcal{\varepsilon}}(\cdot) \geq 0$, it is clear that $\Gamma \neq \varnothing$ and $c_{2} \geq 0$. Moreover, by virtue of (4.3), we have

$$
\sup _{y \in \partial K / \varepsilon} I_{\varepsilon} \circ \gamma \leq S_{p}\left(1, \underline{c}+\delta, \mathbb{R}^{N}\right)+\frac{\eta}{4} \quad \forall \gamma \in \Gamma
$$

Here we claim the following fact.

Claim 4.2.

$$
\forall \gamma \in \Gamma, \quad \exists y_{\varepsilon, \gamma} \in \frac{K}{\varepsilon}: \beta_{R} \circ \gamma\left(y_{\varepsilon, \gamma}\right)=0
$$

Proof of Claim 4.2. For all $\gamma \in \Gamma$, there exists $y_{\varepsilon, \gamma} \in K / \varepsilon$ such that $\beta_{R} \circ \gamma\left(y_{\varepsilon, \gamma}\right)=0$.

$$
F\left(t, y_{\varepsilon}\right) \neq 0 \quad \forall\left(t, y_{\varepsilon}\right) \in[0,1] \times \frac{\partial K}{\varepsilon}
$$

Suppose on the contrary there exists $\left(\bar{t}, \bar{y}_{\varepsilon}\right) \in[0,1] \times \partial K / \varepsilon$ such that $F\left(\bar{t}, \bar{y}_{\varepsilon}\right)=0$. Then by (4.6) we have $\bar{y}_{\varepsilon} \neq 0$ and, in view of (4.2) and (4.3), $\beta_{R} \circ \Phi_{\varepsilon, \underline{c}+\delta}\left(y_{\varepsilon}\right) \in B(0, s)^{c}$. Hence we deduce that $\bar{t} \neq 0,1$ and $\beta_{R} \circ \Phi_{\varepsilon, \underline{c}+\delta}\left(\bar{y}_{\varepsilon}\right)=-\bar{t} \bar{y}_{\varepsilon} /(1-\bar{t})$.

But this is impossible because of (4.4) and (4.5). Therefore (4.14) holds.

Property (4.15) and the homotopy invariance of the topological degree imply that

$$
1=\operatorname{deg}\left(\mathrm{id}, \frac{K}{\varepsilon}, 0\right)=\operatorname{deg}\left(\beta_{R} \circ \gamma(\cdot), \frac{K}{\varepsilon}, 0\right) .
$$

Therefore there exists $y_{\varepsilon, \gamma} \in K / \varepsilon$ such that $\beta_{R} \circ \gamma\left(y_{\varepsilon, \gamma}\right)=0$.

Now we go back to the proof of the second part of Claim 4.1.

By virtue of (4.14) and (4.2), it follows that

$$
\max _{y \in K / \mathcal{\varepsilon}} I_{\varepsilon} \circ \gamma(y) \geq I_{\varepsilon} \circ \gamma\left(y_{\varepsilon, \gamma}\right)=I_{\varepsilon} \circ \Phi_{\varepsilon, \underline{c}+\delta}\left(y_{\varepsilon, \gamma}\right) \geq S_{p}\left(1, \underline{c}+\delta, \mathbb{R}^{N}\right)+\eta \quad \forall \gamma \in \Gamma,
$$

which means

$$
c_{2}=\inf _{\gamma \in \Gamma} \max _{y \in K / \varepsilon} I_{\varepsilon} \circ \gamma(y) \geq S_{p}\left(1, \underline{c}+\delta, \mathbb{R}^{N}\right)+\eta
$$

Then (4.13) and (4.18) imply that

$$
c_{2}=S_{p}\left(1, \underline{c}+\delta, \mathbb{R}^{N}\right)+\eta>S_{p}\left(1, \underline{c}+\delta, \mathbb{R}^{N}\right)+\frac{\eta}{4} \geq \sup _{y \in \partial K / \varepsilon} I_{\varepsilon} \circ \gamma(y) .
$$


Hence all the hypothesis of Proposition 3.7 with $M=M_{p}(\Omega / \mathcal{\varepsilon}), \varphi=\Phi_{\varepsilon, \underline{c}+\delta}, A=\mathbb{R}^{N}, A_{0}=$ $K / \varepsilon$, and $c=c_{2}$ is satisfied. Therefore

$$
c_{2} \geq S_{p}\left(1, \underline{c}+\delta, \mathbb{R}^{N}\right)+\eta>c_{1},
$$

and $c_{2}$ is a critical value.

\section{Acknowledgment}

The author would like to express his gratitude to Professor Mitsuharu Ôtani for his continual encouragement and critical reading of the first version of the manuscript.

\section{References}

[1] S. Adachi and K. Tanaka, Four positive solutions for the semilinear elliptic equation: $-\Delta u+u=$ $a(x) u^{p}+f(x)$ in $\mathbf{R}^{N}$, Calc. Var. Partial Differential Equations 11 (2000), no. 1, 63-95.

[2] P. W. Bates, E. N. Dancer, and J. Shi, Multi-spike stationary solutions of the Cahn-Hilliard equation in higher-dimension and instability, Adv. Differential Equations 4 (1999), no. 1, 1-69.

[3] F. Bethuel, H. Brezis, and F. Hélein, Ginzburg-Landau Vortices, Progress in Nonlinear Differential Equations and Their Applications, vol. 13, Birkhäuser Boston, Massachusetts, 1994.

[4] G. Cerami and D. Passaseo, Existence and multiplicity of positive solutions for nonlinear elliptic problems in exterior domains with "rich" topology, Nonlinear Anal. 18 (1992), no. 2, 109119.

[5] S. Cingolani and M. Lazzo, Multiple semiclassical standing waves for a class of nonlinear Schrödinger equations, Topol. Methods Nonlinear Anal. 10 (1997), no. 1, 1-13.

[6] E. N. Dancer, On the uniqueness of the positive solution of a singularly perturbed problem, Rocky Mountain J. Math. 25 (1995), no. 3, 957-975.

[7] M. del Pino and P. L. Felmer, Local mountain passes for semilinear elliptic problems in unbounded domains, Calc. Var. Partial Differential Equations 4 (1996), no. 2, 121-137.

[8] Semi-classical states for nonlinear Schrödinger equations, J. Funct. Anal. 149 (1997), no. $1,245-265$.

[9] M. K. Kwong, Uniqueness of positive solutions of $\Delta u-u+u^{p}=0$ in $\mathbf{R}^{n}$, Arch. Rational Mech. Anal. 105 (1989), no. 3, 243-266.

[10] O. Rey, A multiplicity result for a variational problem with lack of compactness, Nonlinear Anal. 13 (1989), no. 10, 1241-1249.

[11] M. Struwe, Variational Methods. Applications to Nonlinear Partial Differential Equations and Hamiltonian Systems, 3rd ed., Ergebnisse der Mathematik und ihrer Grenzgebiete. 3. Folge. A Series of Modern Surveys in Mathematics, vol. 34, Springer, Berlin, 2000.

[12] M. Willem, Minimax Theorems, Progress in Nonlinear Differential Equations and Their Applications, vol. 24, Birkhäuser Boston, Massachusetts, 1996.

Michinori Ishiwata: Department of Mathematical Sciences, School of Science and Engineering, Waseda University, 3-4-1 Okubo Shinjuku-ku, Tokyo 169-8555, Japan

E-mail address: ishiwata@otani.phys.waseda.ac.jp 


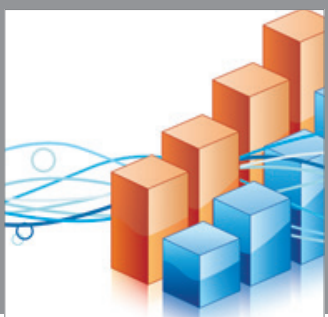

Advances in

Operations Research

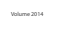

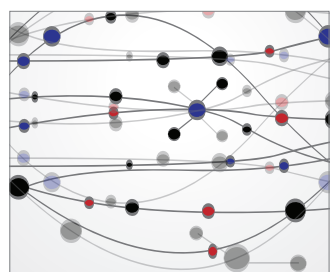

\section{The Scientific} World Journal
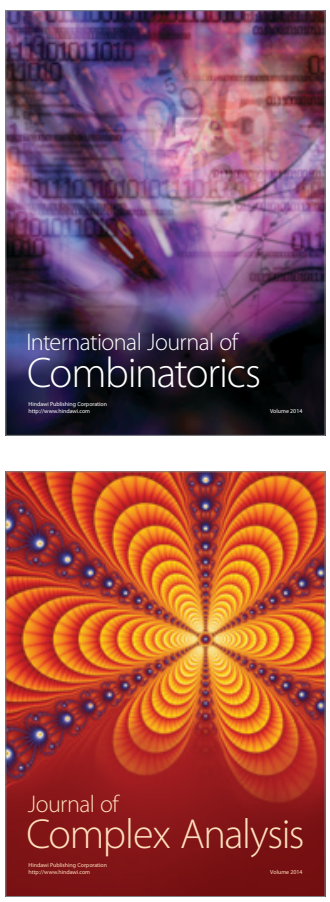

International Journal of

Mathematics and

Mathematical

Sciences
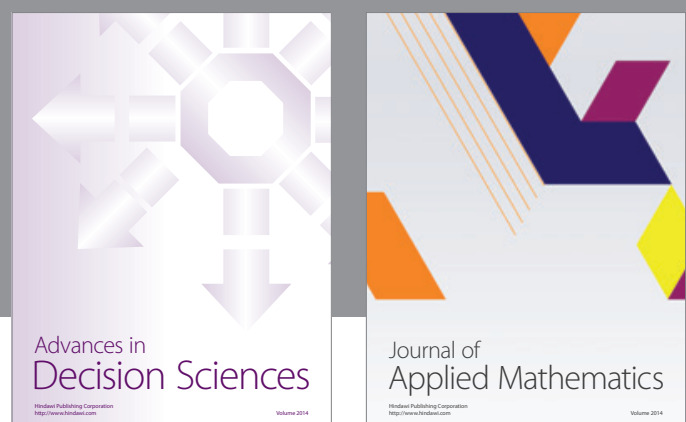

Journal of

Applied Mathematics
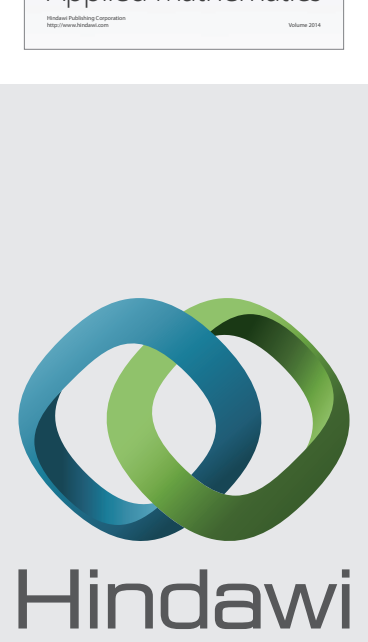

Submit your manuscripts at http://www.hindawi.com
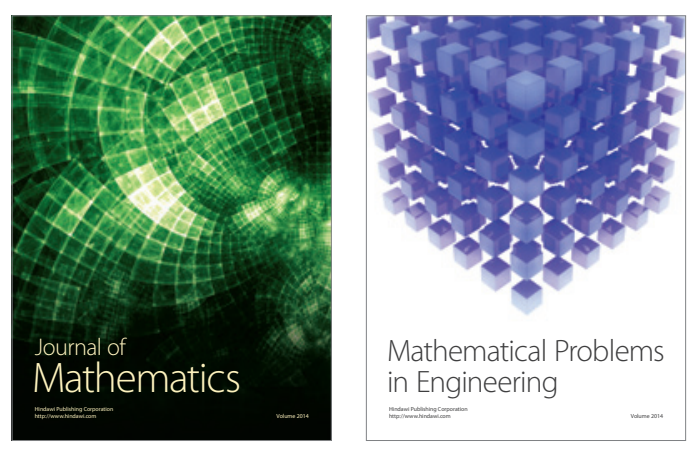

Mathematical Problems in Engineering
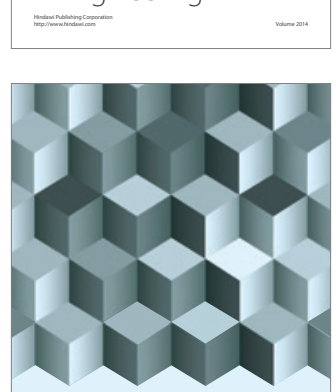

Journal of

Function Spaces
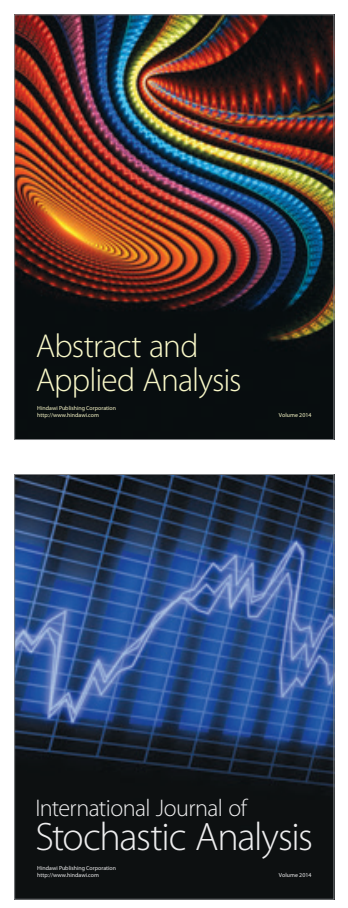

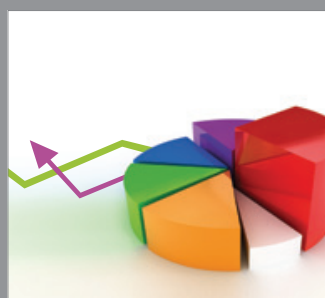

ournal of

Probability and Statistics

Promensencen
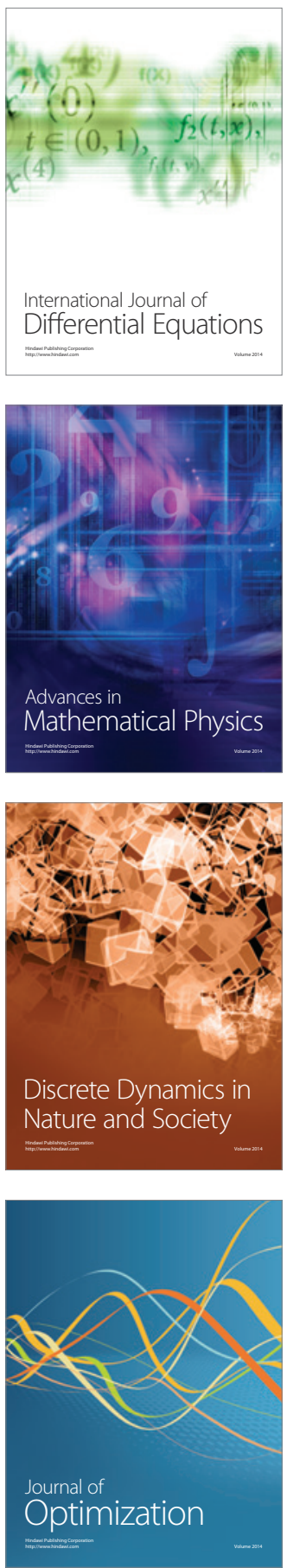